\title{
Glycemic Control and Its Relationship with Diabetes Self-Care Behaviors Among Patients with Type 2 Diabetes in Northern Jordan: A Cross-Sectional Study
}

\author{
Maysa H Almomani $\mathbb{I}^{\prime}$, Shahinaz AL-Tawalbeh² \\ 'Department of Adult Health Nursing, Faculty of Nursing, Jordan University of Science and Technology, Irbid, Jordan; ${ }^{2}$ Department of Community and \\ Mental Health Nursing, Faculty of Nursing, Jordan University of Science and Technology, Irbid, Jordan \\ Correspondence: Maysa H Almomani, Tel +962 27201000 Ext 26I76, Fax +962 2 7201065, Email mhalmomnai@just.edu.jo
}

Background: Type 2 diabetes mellitus (T2DM) is a chronic progressive disease that significantly impacts morbidity and mortality worldwide. Glycemic control is central in diabetes management, yet complex and challenging to achieve; it can be affected by various factors. Diabetes self-care behaviors are vital strategies for effective T2DM management.

Purpose: To evaluate the levels of glycemic control and diabetes self-care behaviors, and the significance of each behavior on glycemic control among patients with T2DM in Jordan.

Patients and Methods: This cross-sectional study was conducted in medical and endocrinology outpatient clinics of three hospitals in northern Jordan. A convenience sample of 520 patients with T2DM participated in the study. Diabetes self-care behaviors, including diet, exercise, blood glucose testing, taking medication, and foot care behaviors, were measured using the Arabic version of the Summary of Diabetes Self-Care Activities (SDSCA-Arabic) questionnaire. Psychological distress was measured using the Arabic version of the 4-items Patient Health Questionnaire (PHQ-4). Glycated hemoglobin (HbAlc) levels were used to assess glycemic control.

Results: Out of 520 participants, $92.7 \%(n=482)$ had poor glycemic control $(\mathrm{HbAlc} \geq 7 \%)$, with a mean of $9.29 \%(\mathrm{SD}=2.06)$ for HbA1c. The SDSCA overall mean score was 3.25 days $(S D=0.88)$, indicating an unsatisfactory level, with $79.2 \%$ of participants having performed self-care activities 4-days/week. Greater adherence to diabetes self-care behaviors was associated with better glycemic control. Exercise was the most important predictor of HbAlc $(\mathrm{p}<0.001)$, followed by general diet $(\mathrm{p}<0.001)$, specific diet $(\mathrm{p}<0.001)$, and blood sugar testing $(\mathrm{p}<0.001)$. Moreover, the Body Mass Index $(\mathrm{p}<0.001)$, treatment type $(\mathrm{p}<0.001)$, and income $(\mathrm{p}=0.03)$ were significant predictors.

Conclusion: The proportions of poor glycemic control and unsatisfactory self-care behaviors were high among T2DM patients. To enhance patients' self-care behaviors and lifestyles changes, the patients need tailored diabetes self-care management educational and supportive programs, conducted by qualified diabetes educators using culturally sensitive strategies.

Keywords: type 2 diabetes mellitus, glycemic control, self-care management, psychological stress, Jordan

\section{Introduction}

Diabetes mellitus (DM) is a significant health concern worldwide that significantly impacts morbidity and mortality. ${ }^{1}$ The global prevalence of diabetes in adults (20-79 years) is high and constantly rising. Diabetes mellitus is expected to increase from 463 million (9.3\%) in 2019 to 578 million (10.2\%) in 2030 and 700 million (10.9\%) in 2045, from whom $79.4 \%$ are living in low- and middle-income countries. ${ }^{2}$ In Jordan, diabetes prevalence was estimated to be $23.7 \%$ in 2017 among people aged $\geq 25$ years. ${ }^{3}$ Type 2 DM (T2DM) accounts for $90-95 \%$ of all diabetes. ${ }^{1,2}$ Diabetes is among the top ten causes of death, resulting in 4.2 million deaths globally in $2019 .^{2}$

High blood glucose level (hyperglycemia) is a major risk factor for many serious life-changing or life-threatening complications including neuropathy, retinopathy, nephropathy, cardiovascular disease, peripheral arterial disease, and 
foot ulceration. ${ }^{4-7}$ Intensive glycemic management is critical in preventing and delaying diabetes complications and related deaths. ${ }^{2,7}$ Unfortunately, poor control of diabetes is widely prevalent worldwide. ${ }^{8-18}$ Different rates of poorly controlled T2DM were revealed in various countries, such as 49.4-74.9\% in Saudi Arabia, ${ }^{19,20} 54 \%$ in Oman, ${ }^{21} 69.6 \%$ in Libya, ${ }^{11} 67.5 \%$ in Turkey, ${ }^{4} 64.1-70.9 \%$ in Ethiopia, ${ }^{13,22} 69.7 \%$ in Tanzania, ${ }^{23} 61.3 \%$ in Zambia, ${ }^{24} 72.6-83 \%$ in Pakistan, ${ }^{8,9} 40 \%$ in Malaysia, ${ }^{25} 89 \%$ in China, ${ }^{10}$ and $66 \%$ in the United States. ${ }^{26}$ In Jordan, poorly controlled T2DM ranged from $56.5 \%$ to $83.8 \%{ }^{15,16,18}$

Diabetes self-care behaviors are key strategies for effective T2DM management, requiring patients' compliance to a healthy diet, regular physical activity, blood sugar testing (BST), foot care, and treatment regimen to achieve optimal glycemic control. ${ }^{2,27}$ Adherence to self-care behaviors is complex and challenging, especially among patients with financial constraints who may not afford diabetes supplies, such as meter testing strips, medications, or healthy foods, ${ }^{26,28}$ leading to a failure in achieving optimal glycemic control. Successful self-care behaviors require patients to perform lifestyle modifications on a daily basis, which may be difficult. ${ }^{29}$ Poor compliance to T2DM self-care behaviors was reported nationally, ${ }^{16,18,29}$ and internationally. ${ }^{11,12,21,30,31}$ Self-management support is one of the six key elements of the chronic care model (CCM) to improve diabetes control in primary care settings. ${ }^{32}$ The significance of self-care management corresponds with many studies' findings, where greater adherence to each self-care behavior, including exercising, ${ }^{8,10,11,18,33}$ dieting, ${ }^{8,16,18,33} \mathrm{BST}^{8}$ and medication adherence, ${ }^{10,23,24,26,33,34}$ is a predictor of better glycemic control. However, several studies could not detect the contribution of self-care behaviors, ${ }^{35,36}$ such as exercising, ${ }^{23,24,26}$ diet care, ${ }^{10,11,23,26}$ BST, ${ }^{10,18,23,24,26,33}$ medication adherence, ${ }^{18}$ and foot care, ${ }^{10,11,18,26,33}$ in predicting glycemic control.

Glycemic control is complex and challenging to achieve because it could also be affected by various sociodemographic, clinical, and psychological factors. Poor glycemic control is associated with older age, ${ }^{10,17}$ female gender, ${ }^{11,13,24}$ low-income, ${ }^{17,37}$ low-education level, unemployment status, ${ }^{11,22}$ obesity, longer duration of diabetes, ${ }^{4,19,23}$ and using insulin treatment (alone or combined with Oral Hypoglycemic Agents [OHA]). ${ }^{10,11,13,17,35}$ Furthermore, high psychological distress was significantly associated with poor glycemic control, ${ }^{10,25,37-39}$ and inadequate diabetes self-care behaviors. $^{9,40}$

There is limited research on the impact of each self-care behavior on glycemic control over other sociodemographic, clinical, and psychological factors among T2DM patients in Jordan. ${ }^{16-18,41,42}$ This study evaluated the levels of glycemic control and diabetes self-care behaviors, and the independent significance of each diabetes self-care behavior for glycemic control among patients with T2DM in Jordan, after controlling for selected sociodemographic, clinical, and psychological factors. Identifying the contribution of each self-care behavior towards glycemic control among patients with T2DM is especially useful in policy-making and prioritizing diabetes self-care interventions to enhance glycemic control.

\section{Materials and Methods}

\section{Research Design, Setting, and Sample}

This cross-sectional correlation study was conducted between April and August 2017 in the medical and endocrinology outpatient clinics of three governmental hospitals in northern Jordan: Princess Basma Hospital (BH), Al-Ramtha Hospital $(\mathrm{RH})$, and Al-Yarmouk hospital (YH). These hospitals provide care for patients with T2DM and are the leading referral hospitals for the north of Jordan.

\section{Sample and Sampling Technique}

A convenience sampling technique was used to recruit patients who visited the outpatient clinics for follow-up care and are diagnosed with T2DM for one year or longer to ensure they had adequate time to experience diabetes self-care activities. Participants included in the study were $\geq 18$ years old, Arabic speakers, and able to take care of themselves. Patients were excluded from the study if they were: 1) pregnant women and 2) with serious physical, mental, or cognitive limitations.

The sample size was calculated based on the number of independent variables required for multiple regression analysis, such as an a priori participant: predictor ratio of (20:1) was used as a rule-of-thumb. ${ }^{43}$ Accordingly, the intended 
sample size was 460 participants; however, the sample was increased by $15 \%$ to account for a possible non-response rate or incomplete records. Thus, the required sample size was 529.

\section{Instruments}

\section{Sociodemographic and Clinical Data}

The questionnaire includes sociodemographic, clinical data based on the literature, ${ }^{8-18}$ The sociodemographic data include age, gender, marital status, level of education, household monthly income, household size (family members residing in the same home), and employment status. The clinical data include health insurance, weight, height, body mass index (BMI), smoking, duration of T2DM, type of antidiabetic treatment, previous education regarding T2DM, comorbidities, glycated hemoglobin ( $\mathrm{HbAlc}$ ), having psychological support from significant others, and diabetes-care assistance from family members.

Weight and height were measured using calibrated equipment and standardized techniques. Weight was measured in light clothing and without footwear using a standard weighing scale to the nearest 0.1 kilograms. Height was measured without footwear to the nearest 0.1 centimeters $(\mathrm{cm})$ using a stadiometer. The BMI was computed using the standard formula (weight in kilograms/(height in meters) ${ }^{2}$ ) and classified according to the World Health Organization guidelines. ${ }^{44}$ Participants' most recent HbA1c results, during the last three months, were obtained from medical records at the point of enrollment in the study and used to measure glycemic control. HbA1c was analyzed at Ministry of Health hospitals using a Chemistry Auto-analyzer with Roche/Hitachi kit after minimum fasting of 8 hours. According to the American Diabetes Association guidelines, HbA1c levels were classified into good glycemic control when HbA1c $<7 \%$ $(<53 \mathrm{mmol} / \mathrm{mol})$ and poor glycemic control when HbAlc $\geq 7.0 \%(\geq 53 \mathrm{mmol} / \mathrm{mol}){ }^{45}$

\section{Psychological Distress}

Participants' psychological distress was assessed using the 4-items Patient Health Questionnaire (PHQ-4), which is a valid ultra-brief tool for screening both anxiety and depressive disorders. The PHQ-4 combines two validated screeners: the 2-item Generalized Anxiety Disorder Screener (GAD-2), which focuses on two core criteria for anxiety, and the 2-item Patient Health Questionnaire (PHQ-2), which focuses on the loss of interest and depressed mood core criteria for depression. ${ }^{46}$ Responses were scored on a 4-point Likert scale of 0 (not at all), 1 (several days), 2 (more than half the days), and 3 (nearly every day) over the past two weeks. Therefore, the PHQ-4 total score ranged from 0 to 12 and served as a general marker of psychological distress, with categories of psychological distress as none (0-2), mild (3-5), moderate (6-8), and severe (9-12). ${ }^{46}$ This study used a validated Arabic version of the PHQ-4 (available at: https://www.phqscreeners.com/select-screener), with good internal consistency for PHQ-4 (Cronbach's alpha $(\alpha)$ $=0.73),{ }^{47}$ which is comparable to that of the original English version $(\alpha=0.82-0.85) .{ }^{46,48}$ In our study, Cronbach's alpha was 0.70 .

\section{Diabetes Self-Care Behaviors}

Diabetes self-care behaviors were assessed using the Arabic version of the Summary of Diabetes Self-Care Activities (SDSCA-Arabic) questionnaire, ${ }^{49,50}$ a translated version of the SDSCA developed by Toobert et al. It consisted of 15 items that assessed the five domains of diabetes self-care activities, including diet (5-items: 2-items on the general diet, 3 -items on the specific diet), exercise (2-items), blood glucose testing (2-items), medication (2-items), and foot care (4-items). The SDSCA-Arabic used an 8-point Likert scale from 0 (none of the days) to 7 days (daily), representing the self-reported frequency of performing diabetes self-care activities during the past week. Item-5 on the dietary subscale assessed the consumption of a high-fat diet, so it was reverse-coded to reflect the adherence to a low-fat diabetes regimen to be scored accordingly. ${ }^{51}$ The mean score for each subscale was calculated in this study according to the original SDSCA guidelines51 to permit comparability with other studies. ${ }^{12,18,20,29,52}$ Then, by averaging subscales mean scores, the overall mean SDSCA-Arabic score also ranged between (0-7) days. Higher scores indicated better self-care behaviors. As recommended, scores between (0-4) days were considered unsatisfactory, and between (5-7) were satisfactory. ${ }^{31}$ The SDSCA-Arabic was valid and reliable $(\alpha=0.76$; test-retest, $r=0.912$; and split-half $=0.90) .{ }^{49}$ In our study, Cronbach's alpha was 0.73 . 


\section{Ethical Considerations}

Ethical approvals were obtained from the Institutional Review Board (IRB) at the Jordan University of Science and Technology and the Jordanian Ministry of Health. Permissions to conduct the study were obtained from the directors of the selected hospitals. Each participant signed a written consent form after explaining the purpose, risks, benefits, and study method. Participation was voluntary, and participants had the right to withdraw at any time without any consequences. Participants were ensured regarding confidentiality of their data by keeping it in a closed cabinet accessible only to the researchers. This study was conducted in accordance with the Declaration of Helsinki.

\section{Data Collection Procedures}

Data were obtained from medical records and by interviewing participants in the selected hospitals. The researchers approached patients with T2DM who met the inclusion and exclusion criteria and invited them to participate in the study after explaining the purpose of the study, risks and benefits of participation, voluntary participation, and their right to withdraw, as well as answering any questions concerning the study. Researchers asked participants who agreed to participate to sign the consent form. Then, one of the two researchers conducted the questionnaire structured face-to-face interview on the same day of their visit in the waiting room. A face-to-face interview was used to ensure that all questions were answered, since many participants cannot read and write. Each interview took about 15-20 minutes to complete. After completing the interview, the second researcher measured each participant's height and weight. Participants' medical records were reviewed to verify the accuracy of reported clinical data, obtain the HbA1c results in the previous three months, and record it in a specific checklist within the utilized instrument. Both researchers doublechecked the completeness of the collected data. Some interviews were interrupted by calling the participant for treatment, causing them to withdraw from the interview.

\section{Statistical Analysis}

Data were analyzed using IBM SPSS 23 (IBM Corp. Released 2015. IBM SPSS Statistics for Windows, Version 23.0. Armonk, NY: IBM Corp). Descriptive statistics of means, standard deviations, frequencies, and percentages were used to describe the participants' data. In addition, independent-sample $t$-tests, one-way analysis of variance (ANOVA) test, and Pearson's correlation coefficients were used to assess the bivariate association between HbAlc levels and sociodemographic, clinical, psychological variables, and diabetes self-care behaviors. Hierarchical multiple linear regression analysis, used by similar studies, ${ }^{35,53}$ was performed to assess the contribution of diabetes self-care behaviors on glycemic control (HbAlc levels) after controlling other confounding variables. Variables with a p-value less than 0.25 in the bivariate analyses were included in the multivariate model. Preliminary analyses were run to ensure that the assumptions of normality, linearity, homoscedasticity, and multicollinearity were not violated. Categorical variables were dummy coded for regression analysis. Sociodemographic, clinical, and psychological variables were entered in step one, and diabetes self-care behaviors were entered in step two. This study had a total of 520 participants and nineteen independent predictor variables, resulting in a participant: predictor ratio of (27:1), which exceeds the minimum required ratio of $(20: 1) .{ }^{43}$ Statistical significance was set at $\mathrm{p}<0.05$.

\section{Results}

\section{Participants' Characteristics: Sociodemographic and Clinical Characteristics}

Out of the eligible 650 participants, 520 patients responded and completed the questionnaire structured interviews, resulting in a response rate of (80\%). Table 1 represents the participants' sociodemographic, clinical, and psychological characteristics. Participants' mean age was 55.9 years $(\mathrm{SD}=11.8, \mathrm{R}=25-90)$, nearly half of them $(51 \%)$ were $\geq 55$ years old. Among the participants, $51 \%$ were males, $77.2 \%$ had secondary education or less, $68.1 \%$ were unemployed, and only $21.5 \%$ were current smokers. The average household monthly income was 312.8 ( $\mathrm{SD}=188$ ) Jordan Dinars (JDs); $80 \%$ of the participants had an income to household size ratio of $68.63(\mathrm{SD}=54.46)$ JDs per household member.

The mean duration of diabetes was 8.1 years ( $\mathrm{SD}=6.38$ ), with $51 \%$ of participants having diabetes for more than five years. Among participants, $64.6 \%$ were using OHA alone, $26.9 \%$ were on insulin alone, and $8.5 \%$ were on a combination 
Table I Participants' Sociodemographic, Clinical, and Psychological Characteristics $(n=520)$

\begin{tabular}{|c|c|}
\hline Characteristics & $\mathbf{N}(\%)$ \\
\hline Age, years, mean (SD) & $55.86(11.8)$ \\
\hline 25-35 Years & $25(4.8)$ \\
\hline 36-45 Years & $80(15.4)$ \\
\hline 46-55 Years & $159(30.6)$ \\
\hline 56-65 Years & 149 (28.7) \\
\hline$>65$ Years & $107(20.6)$ \\
\hline \multicolumn{2}{|l|}{ Gender } \\
\hline Female & 255(49) \\
\hline Male & $265(5 I)$ \\
\hline \multicolumn{2}{|l|}{ Marital status } \\
\hline Single & $25(4.8)$ \\
\hline Currently married & 405(77.9) \\
\hline Widow & $79(15.2)$ \\
\hline Divorce & $\mathrm{II}(2 . \mathrm{I})$ \\
\hline \multicolumn{2}{|l|}{ Educational level } \\
\hline Illiterate & $97(18.7)$ \\
\hline Primary & $170(32.7)$ \\
\hline Secondary & $134(25.8)$ \\
\hline Diploma & $54(10.4)$ \\
\hline Bachelor's Degree & $52(10)$ \\
\hline Higher education. & $13(2.5)$ \\
\hline \multicolumn{2}{|l|}{ Employment status } \\
\hline No & $354(68.1)$ \\
\hline Yes & 166(31.9) \\
\hline Household monthly income, JD's, mean (SD) & $312.8(188)$ \\
\hline Household sizes, persons, mean (SD) & $4.8(2.4)$ \\
\hline \multicolumn{2}{|l|}{ Health insurance } \\
\hline Yes. & 432 (83.I) \\
\hline No. & $88(16.9)$ \\
\hline \multicolumn{2}{|l|}{ Smoking status } \\
\hline Yes & $112(21.5)$ \\
\hline No & $408(78.5)$ \\
\hline \multicolumn{2}{|l|}{ Comorbidities } \\
\hline Yes & $275(52.9)$ \\
\hline No & $245(47.1)$ \\
\hline BMI $\left(\mathbf{k g} / \mathbf{m}^{\mathbf{2}}\right)$, mean (SD) & $30.07(5.88)$ \\
\hline Underweight $\left(<18.5 \mathrm{~kg} / \mathrm{m}^{2}\right)$ & $2(0.40)$ \\
\hline Normal weight $\left(18.5-24.9 \mathrm{~kg} / \mathrm{m}^{2}\right)$ & $89(17.1)$ \\
\hline Overweight $(25-29.9$ kg/m²) & $207(39.8)$ \\
\hline Obese $\left(\geq 30 \mathrm{~kg} / \mathrm{m}^{2}\right)$ & $222(42.7)$ \\
\hline Pre-obese-I $\left(30-34.9 \mathrm{~kg} / \mathrm{m}^{2}\right)$ & $133(25.6)$ \\
\hline Obese class-II $(35-39.9$ kg/m²) & $52(10.0)$ \\
\hline Obese class-III ( $\geq 40$ kg/m²) & $37(7.1)$ \\
\hline
\end{tabular}

(Continued) 
Table I (Continued).

\begin{tabular}{|c|c|}
\hline Characteristics & $\mathbf{N}(\%)$ \\
\hline Diabetes duration, years, mean (SD) & 8.1 (6.38) \\
\hline $\mathrm{I}-5$ years & $255(49)$ \\
\hline $6-10$ years & $129(24.8)$ \\
\hline $1 \mathrm{I}-15$ years & $78(15)$ \\
\hline $16-20$ years & $38(7.3)$ \\
\hline$>20$ years & $20(3.8)$ \\
\hline \multicolumn{2}{|l|}{ Treatment types } \\
\hline $\mathrm{OHA}$ alone & $336(64.6)$ \\
\hline Insulin alone & $140(26.9)$ \\
\hline OHA \& insulin & $44(8.5)$ \\
\hline \multicolumn{2}{|l|}{ Previous diabetes education } \\
\hline Yes & $59(11.3)$ \\
\hline No & $46 I(88.7)$ \\
\hline \multicolumn{2}{|l|}{ Diabetes education setting } \\
\hline Clinic & $14(23.7)$ \\
\hline Hospital & $28(47.5)$ \\
\hline UNRWA clinics & $\mathrm{II}(18.6)$ \\
\hline Medicine without Border & $6(10.2)$ \\
\hline \multicolumn{2}{|l|}{ Help with diabetes self-care at home } \\
\hline Yes & 255 (49) \\
\hline No & $265(5 \mathrm{I})$ \\
\hline \multicolumn{2}{|l|}{ Psychological support } \\
\hline Yes & $430(82.7)$ \\
\hline No & $90(17.3)$ \\
\hline HbAlc, \%, mean (SD) & $9.29(2.06)$ \\
\hline \multicolumn{2}{|l|}{ Glycemic control status } \\
\hline Good control $<7 \%$ & $38(7.3 \%)$ \\
\hline Poor control $\geq 7 \%$ & $482(92.7 \%)$ \\
\hline
\end{tabular}

Notes: Data presented as $\mathrm{n}$ (\%) or mean and standard deviation (SD); Household size= the number of family members who live in the same home.

Abbreviations: JD's, Jordan dinars; BMI, body mass index; OHA, oral hypoglycemic agents; HbAlc, glycated hemoglobin.

of OHA and insulin therapy. About $52.9 \%$ of the participants had comorbidities, such as hypertension, cardiac disease, renal impairments, vision impairments, and peripheral neuropathy. Participants' mean BMI was 30.07 (SD=5.88), 42.7\% were obese and 39.8\% were overweight. Participants had high means of HbAlc (M=9.29\%, SD=2.06); 92.7\% had poor glycemic control. Only $11.3 \%$ of participants attended diabetes education sessions, mainly at hospitals (47.5\%). Most participants (82.7\%) had psychological support when needed, while only $49 \%$ of participants had direct diabetes-care help from family members.

\section{Psychological Distress}

The mean overall PHQ-4 score of the psychological distress score was 5.51 ( $\mathrm{SD}=3.83$ ) out of 12, the maximum, denoting a generally moderate level of psychological distress, according to the classification cutoffs devised by the PHQ-4 authors. ${ }^{46}$ Overall, it is noticeable that $30 \%$ had reported moderate levels of psychological distress, and an approximately equal proportion $(29 \%$, ) had mild distress, while $17.5 \%$ reported severe distress (see Table 2). 
Table 2 Participants' Psychological Distress

\begin{tabular}{|l|l|l|}
\hline PHQ-4 Items & Mean (SD) & N (\%) \\
\hline Little interest or pleasure in doing things & $1.31(1.02)$ & \\
Feeling down, depressed, or hopeless & $1.09(1.34)$ & \\
Feeling nervous, anxious or on edge & $1.50(1.75)$ & \\
Not being able to stop or control worrying & $1.59(1.06)$ & \\
\hline Total score & $5.51(3.83)$ & $122(23.5)$ \\
None (0-2) & & $151(29)$ \\
Mild distress (3-5) & & $156(30)$ \\
Moderate distress (6-8) & & $91(17.5)$ \\
Severe distress (9-12) & & \\
\hline
\end{tabular}

Abbreviations: PHQ-4, the 4-items Patient Health Questionnaire; SD, standard deviation.

\section{Adherence to Self-Care Activities}

Table 3 shows the mean scores of SDSCA, representing the mean number of days during the past seven days the participants performed diabetes self-care activities. The overall mean score for SDSCA was 3.25 days (SD=0.88), indicating an unsatisfactory level of self-care behaviors, with $79.2 \%$ of participants having performed self-care activities 4-days/week. The top-rated domain of SDSCA was taking medication with a mean of 6.33 days $(\mathrm{SD}=1.45)$ per week, which was the only satisfactory level activity. Exercising and blood-sugar testing were the bottom-rated domains with a means of $1.05(\mathrm{SD}=1.19)$ and $1.52(1.58)$ days, respectively (see Table 3). Overall, the top reported self-care activities were taking insulin $(\mathrm{M}=6.50, \mathrm{SD}=1.34)$ and $\mathrm{OHA}(\mathrm{M}=6.17, \mathrm{SD}=1.65)$, washing feet $(\mathrm{M}=5.69, \mathrm{SD}=2.21)$, and eating five or more servings of vegetables $(\mathrm{M}=5.10, \mathrm{SD}=1.97)$. The lowest-rated self-care activities were participating in a specific exercise session $(\mathrm{M}=0.18, \mathrm{SD}=0.56)$ and testing blood sugar levels as recommended $(\mathrm{M}=0.94, \mathrm{SD}=01.43)$.

\section{Sociodemographic, Clinical, Psychological Factors, and Self-Care Behaviors Relationship with Glycemic Control}

As shown in Table 4, HbA1c levels were significantly lower among participants with higher household income ( $(\mathrm{r}(518)=$ $-0.254, \mathrm{p}<0.001)$, higher education levels $(\mathrm{F}(5514)=5.91, \mathrm{p}<0.001)$, and an employed status, $\mathrm{t}(518)=3.18, \mathrm{p}=0.002$. Also, some clinical characteristics had a significant association with HbAlc. Participants' HbAlc levels had significant positive correlations with their BMI ( $\mathrm{r}(518)=0.33, \mathrm{p}<0.001)$ and diabetic disease duration, but weakly, $(\mathrm{r}(518)=0.13$, $\mathrm{p}=0.003)$. Also, participants receiving various diabetic treatments had significantly different HbA1c levels, F (2517) = 8.26, $\mathrm{p}<0.001$. A follow-up post-hoc pairwise comparison showed that patients taking only OHA had significantly lower HbA1c than those undertaking insulin treatment, $\mathrm{p}<0.001$. Moreover, the pairwise comparisons indicated that those taking mixed treatments do not differ statistically from those taking insulin alone or diabetic tablets alone (post-hoc results not included in the tables).

A greater sense of psychological distress was significantly, but weakly, associated with higher HbA1c levels; $r=0.133$, $\mathrm{p}=0.003$. However, those who obtained psychological support had significantly lower HbA1c scores than those who did not, $\mathrm{t}(518)=2.72, \mathrm{p}=0.007$ (see Table 4). Moreover, lower HbAlc level was significantly associated with greater compliance to diabetes self-care behaviors, including general dietary $(\mathrm{r}(518)=-0.422, \mathrm{p}<0.001)$, specific dietary $(\mathrm{r}(518)$ $=-0.362, \mathrm{p}<0.001)$, exercise $(\mathrm{r}(518)=-0.577, \mathrm{p}<0.001)$, BST $(\mathrm{r}(518)=-0.315, \mathrm{p}<0.001)$, and foot self-care behaviors $(\mathrm{r}(518)=-0.203, \mathrm{p}<0.001)$. Surprisingly, the treatment-taking frequency had no significant association with higher or lower HbA1c levels (see Table 3). 
Table 3 Diabetes Self-Care Activities and Its Correlation with HbAlc $(n=520)$

\begin{tabular}{|c|c|c|c|c|}
\hline SDSCA Subscales & Items & $\begin{array}{c}\text { Mean (SD) } \\
\text { Days/Week }^{\mathrm{a}}\end{array}$ & $\begin{array}{l}\text { Correlation } \\
\text { with HbAlc }\end{array}$ & p-value \\
\hline $\begin{array}{l}\text { Diet } \\
\text { General diet } \\
\text { Specific diet }\end{array}$ & $\begin{array}{l}\text { I. Follow a healthy eating plan in the last week } \\
\text { 2. Follow your eating plan over the past month } \\
\text { 3. Eat } 2-3 \text { servings of fruits } \\
\text { 4. Eat five or more servings of vegetables } \\
\text { 5. Eat high-fat foods such as red meat or whole-fat dairy products } \\
\text { (Reverse scoring item) }\end{array}$ & $\begin{array}{l}3.71(1.42) \\
3.4(2.41) \\
3.32(2.50) \\
3.48(2.47) \\
3.91(1.45) \\
3.87(2.08) \\
5.10(1.97) \\
2.75(2.1)\end{array}$ & $\begin{array}{l}-0.50 \\
-0.422\end{array}$ & $\begin{array}{l}<0.001 \\
<0.001 \\
<0.001\end{array}$ \\
\hline Exercise & $\begin{array}{l}\text { 6. Participate in at least } 30 \text { minutes of physical activity (Total minutes of } \\
\text { continuous activity, including walking) } \\
\text { 7. Participate in a specific exercise session (such as walking and biking) }\end{array}$ & $\begin{array}{l}1.05(1.19) \\
1.93(2.26) \\
0.18(0.56)\end{array}$ & -0.577 & $<0.001$ \\
\hline $\begin{array}{l}\text { Blood sugar } \\
\text { testing }\end{array}$ & $\begin{array}{l}\text { 8. Test for blood sugar } \\
\text { 9. Test blood sugar the number of times recommended by your health } \\
\text { care provider }\end{array}$ & $\begin{array}{l}1.52(1.58) \\
2.1(2.16) \\
0.94(1.43)\end{array}$ & -0.315 & $<0.001$ \\
\hline Foot care & $\begin{array}{l}\text { 10. Checked your feet } \\
\text { II. Inspect the inside of your shoes } \\
\text { 12. Wash your feet } \\
\text { 13. Dry between your toes after washing }\end{array}$ & $\begin{array}{l}3.66(2.08) \\
2.93(2.98) \\
2.58(2.93) \\
5.96(2.21) \\
3.16(3.1)\end{array}$ & -0.203 & $<0.001$ \\
\hline Medication & $\begin{array}{l}\text { 14. Inject insulin according to recommended doses }(n=184) \\
\text { 15. Take OHA at the recommended dosage }(n=380)\end{array}$ & $\begin{array}{l}6.33(1.45) \\
6.49(1.34) \\
6.17(1.65)\end{array}$ & 0.019 & 0.663 \\
\hline $\begin{array}{l}\text { Overall mean } \\
\text { score }\end{array}$ & $\begin{array}{l}\text { Unsatisfactory }(<4): 79.2 \% \text {. } \\
\text { Satisfactory level }(\geq 4): 20.8 \% \text {; }\end{array}$ & $3.25(0.88)$ & -0.168 & $<0.001$ \\
\hline
\end{tabular}

Notes: a Scale scores range: 0-7; Test of association: Pearson's correlation coefficients (r). Adapted with permission from Toobert DJ, Hampson SE, Glasgow RE. The summary of diabetes self-care: Results from 7 studies and a revised scale. Diabetes Care. 2000;23(7):943-950. SDSCA Copyright 2000 Oregon Research Institute, Eugene, Oregon. All rights reserved. ${ }^{51}$

Abbreviations: SDSCA, Summary of Diabetes Self-Care Activities; HbAlc, glycated hemoglobin; SD, standard deviation; OHA, oral hypoglycemic agents.

\section{The Contribution of Diabetes Self-Care Behaviors in Predicting Glycemic Control Over Sociodemographic, Clinical, and Psychological Variables}

As shown in Table 5, a two-step hierarchical multiple regression analysis was performed to assess the contribution of each diabetes self-care behavior in glycemic control prediction, controlling the potential confounders included in this study. Preliminary analyses were run to ensure that the assumptions of normality, linearity, homoscedasticity, and multicollinearity were not violated. Sociodemographic, clinical, and psychological distress variables were entered in model one, and diabetes self-care behaviors in model two. Model one explained $22.3 \%$ of the variance in HbA1c levels, $\mathrm{F}(14,501)=10.25, \mathrm{p}<0.001$. However, only BMI $(\beta=0.297, \mathrm{p}<0.001)$, treatment type $(\beta=0.156, \mathrm{p}<0.001)$, income $(\beta=$ $-0.150, p=0.001)$, educational level $(\beta=-0.138, p=0.002)$, and psychological distress $(\beta=0.083, p=0.041)$ were significant predictors in this regression model. As patients' economic status and educational levels tended to rise, their HbA1c levels tended to decline significantly, after controlling the other variables in the model. Conversely, patients with higher levels of BMI and psychological distress, and with insulin treatment, were significantly associated with higher HbA1c levels. 
Table 4 Bivariate Association Between Participants' Characteristics and HbAlc $(n=520)$

\begin{tabular}{|c|c|c|c|}
\hline Characteristics & HbAlc Mean (SD) & Test of Association & $p$-value \\
\hline $\begin{array}{l}\text { Age, years, } \\
\begin{array}{l}25-35 \text { years } \\
36-45 \text { years } \\
46-55 \text { years } \\
56-65 \text { years } \\
>65 \text { years }\end{array}\end{array}$ & $\begin{array}{l}8.84(2.11) \\
8.93(2.12) \\
9.29(1.71) \\
9.55(2.30) \\
9.32(2.08)\end{array}$ & $\begin{array}{c}0.08^{\mathrm{a}} \\
1.488^{\mathrm{b}}\end{array}$ & $\begin{array}{l}0.069 \\
0.204\end{array}$ \\
\hline $\begin{array}{c}\text { Gender } \\
\text { Female } \\
\text { Male }\end{array}$ & $\begin{array}{l}9.43(9.43) \\
9.16(9.16)\end{array}$ & $1.516^{\mathrm{c}}$ & 0.130 \\
\hline $\begin{array}{l}\text { Marital status } \\
\text { Single } \\
\text { Ever married }\end{array}$ & $\begin{array}{l}8.76(2.07) \\
9.32(2.05)\end{array}$ & $1.32^{\mathrm{c}}$ & 0.188 \\
\hline $\begin{array}{l}\text { Education level } \\
\text { Illiterate } \\
\text { Primary } \\
\text { Secondary } \\
\text { Diploma } \\
\text { Bachelor's Degree } \\
\text { Higher education. }\end{array}$ & $\begin{array}{l}9.70(2.31) \\
9.74(2.14) \\
9.02(1.74) \\
8.74(1.90) \\
8.70(1.72) \\
7.94(1.85)\end{array}$ & $5.91^{\mathrm{b}}$ & $<0.00$ I \\
\hline $\begin{array}{l}\text { Employment status } \\
\text { No } \\
\text { Yes }\end{array}$ & $\begin{array}{c}9.49(2.07) \\
8.88(2.0)\end{array}$ & $3.18^{\mathrm{c}}$ & 0.002 \\
\hline Household monthly income, JD's, & & $-0.254^{\mathrm{a}}$ & $<0.001$ \\
\hline Household sizes, persons & & $-0.025^{\mathrm{a}}$ & 0.569 \\
\hline $\begin{array}{l}\text { Comorbidities } \\
\text { Yes } \\
\text { No }\end{array}$ & $\begin{array}{c}9.39(2.12) \\
9.18(2.0)\end{array}$ & $-1.171^{\mathrm{c}}$ & 0.242 \\
\hline $\begin{array}{l}\text { BMI }\left(\mathbf{k g} / \mathbf{m}^{2}\right) \\
\text { Underweight }\left(<18.5 \mathrm{~kg} / \mathrm{m}^{2}\right) \\
\text { Normal Weight }\left(18.5-24.9 \mathrm{~kg} / \mathrm{m}^{2}\right) \\
\text { Overweight }\left(25-29.9 \mathrm{~kg} / \mathrm{m}^{2}\right) \\
\text { Obese }\left(\geq 30 \mathrm{~kg} / \mathrm{m}^{2}\right) \\
\text { Pre-obese class I } \\
\text { Obese class II } \\
\text { Obese class III }\end{array}$ & $\begin{array}{l}7.50(1.70) \\
8.91(1.74) \\
8.74(1.72) \\
9.64(2.04) \\
9.67(2.04) \\
11.63(2.61)\end{array}$ & $\begin{array}{l}0.329^{a} \\
\left.16.8\right|^{b}\end{array}$ & $\begin{array}{l}<0.001 \\
<0.001\end{array}$ \\
\hline $\begin{array}{l}\text { Diabetes duration, years } \\
\text { I-5 Years } \\
6-10 \text { Years } \\
\text { II-15 Years } \\
16-20 \text { Years } \\
>20 \text { Years }\end{array}$ & $\begin{array}{l}9.08(1.96) \\
9.48(2.02) \\
9.33(2.14) \\
9.33(2.10) \\
10.49(2.66)\end{array}$ & $\begin{array}{l}0.129^{\mathrm{a}} \\
2.68^{\mathrm{b}}\end{array}$ & $\begin{array}{l}0.003 \\
0.031\end{array}$ \\
\hline $\begin{array}{l}\text { Treatment types } \\
\text { OHA alone } \\
\text { Insulin alone } \\
\text { OHA \& insulin }\end{array}$ & $\begin{array}{l}9.03(1.94) \\
9.82(2.27) \\
9.63(1.85)\end{array}$ & $8.26^{\mathrm{b}}$ & $<0.001$ \\
\hline
\end{tabular}

(Continued) 
Table 4 (Continued).

\begin{tabular}{|c|c|c|c|}
\hline Characteristics & HbAlc Mean (SD) & Test of Association & $p$-value \\
\hline \multicolumn{4}{|l|}{ Health insurance } \\
\hline Yes. & $9.31(2.02)$ & $0.529^{c}$ & 0.597 \\
\hline No. & $9.19(2.23)$ & & \\
\hline \multicolumn{4}{|l|}{ Smoking status } \\
\hline Yes & $9.31(2.03)$ & $0.37^{c}$ & 0.772 \\
\hline No & $9.24(2.16)$ & & \\
\hline \multicolumn{4}{|c|}{ Previous diabetes education } \\
\hline Yes & $9.33(2.06)$ & $1.24^{\mathrm{c}}$ & 0.216 \\
\hline No & $8.98(2.0)$ & & \\
\hline \multicolumn{4}{|c|}{ Help with diabetes self-care at home } \\
\hline Yes & $9.43(1.96)$ & $1.67^{c}$ & 0.095 \\
\hline No & $9.44(2.14)$ & & \\
\hline \multicolumn{4}{|l|}{ Psychological support } \\
\hline Yes & $9.18(2.02)$ & $2.72^{\mathrm{c}}$ & 0.007 \\
\hline No & $9.83(2.16)$ & & \\
\hline Psychological distress & & $0.131^{\mathrm{a}}$ & 0.003 \\
\hline
\end{tabular}

Notes: a Pearson's correlation coefficients (r); ${ }^{b}$ One-way analysis of variance; 'Independent sample $t$-test; Household size $=$ the number of family members who live in the same home.

Abbreviations: HbAlc, glycated hemoglobin; SD, standard deviation; JD's, Jordan Dinars; BMI, body mass index; OHA, oral hypoglycemic agents.

Adding diabetes self-care behaviors (except medication) in model two accounted for a significant additional $30.3 \%$ of the variance in the HbAlc level, $\Delta \mathrm{R}^{2}=0.303$, Fchange $(5496)=63.52, \mathrm{p}<0.001$. The total variance explained by model two was $52.6 \%, \mathrm{~F}(19,496)=28.98, \mathrm{p}<0.001$. The exercise was the most important predictor of HbA1c $(\beta=-0.363$, $\mathrm{p}<0.001)$, followed by general diet $(\beta=-0.209, \mathrm{p}<0.001)$, specific $\operatorname{diet}(\beta=-0.153, \mathrm{p}<0.001)$, and $\mathrm{BST}(\beta=-0.143$, $\mathrm{p}<0.001)$.

Model two suggested that only patients' BMI $(\beta=0.174, \mathrm{p}<0.001)$, type of treatment $(\beta=0.149, \mathrm{p}<0.001)$, and income $(\beta=$ $-0.080, \mathrm{p}=0.03$ ) remained significant predictors of $\mathrm{HbA} 1 \mathrm{c}$ levels when considered jointly with diabetes self-care behaviors as predictors. However, educational level and psychological distress were not significant in predicting HbAlc levels.

\section{Discussion}

\section{Glycemic Control Status}

Most of the study participants (92.7\%) had high HbA1c levels, greater than the standard cut-off value (7\%), indicating a failure to meet the American Diabetes Association recommendations. ${ }^{45}$ Poor glycemic control was reported by previous international $^{8-13}$ and local studies. ${ }^{14-18}$ However, this prevalence of poor glycemic control is higher than previously reported rates in Jordan, (56.5\% to 83.8\%), ${ }^{15,16,18}$ other Arabian countries, such as Saudi Arabia (49.4-74.9\%), ${ }^{19,20}$ Oman (54\%), ${ }^{21}$ and Libya (69.6\%), ${ }^{11}$ and non-Arabian countries (40-83\%). ${ }^{4,8,9,13,22-26}$ The high prevalence of poor glycemic control among the participants is alarming, since poor glycemic control among patients with T2DM has serious adverse health and financial consequences, ${ }^{4,54}$ and requires immediate interventions.

\section{Self-Care Behaviors and Their Contribution to Glycemic Control}

Participants' poor glycemic control was significantly associated with unsatisfactory compliance with self-care behaviors. Most study participants reported unsatisfactory compliance to diabetes self-care behaviors, less than four days/week on average, consistent with the findings of previous Jordanian ${ }^{16,18,29}$ and international studies. ${ }^{11,12,21,30,31}$ In contrast, Qteishat \& Ghananim (2015) reported high compliance to diabetes self-care activities using the same measurement tool. However, their 
Table 5 Summary of Hierarchical Regression Analysis for Variables Predicting HbAlc Levels (Glycemic Control)

\begin{tabular}{|c|c|c|c|c|c|c|c|c|c|c|}
\hline \multirow[t]{2}{*}{ Predictors } & \multicolumn{5}{|c|}{ Step I } & \multicolumn{5}{|c|}{ Step 2} \\
\hline & B & SE & $\beta$ & $\mathbf{t}$ & p-value & B & SE & $\boldsymbol{\beta}$ & $\mathbf{t}$ & p-value \\
\hline (Constant) & 7.404 & 0.763 & & 9.70 & $<0.001$ & 9.919 & 0.629 & & 15.8 & $<0.001$ \\
\hline Age (years) & -0.004 & 0.009 & -0.024 & -0.47 & 0.640 & 0.001 & 0.007 & 0.008 & 0.20 & 0.844 \\
\hline Gender (male) & -0.253 & 0.185 & -0.061 & -1.36 & 0.174 & -0.134 & 0.147 & -0.032 & -0.91 & 0.365 \\
\hline Marital status (ever married) & 0.222 & 0.401 & 0.023 & 0.56 & 0.579 & 0.070 & 0.315 & 0.007 & 0.22 & 0.824 \\
\hline Education level ( $\geq$ secondary) & -0.568 & 0.186 & -0.138 & -3.05 & 0.002 & -0.076 & 0.151 & -0.018 & -0.50 & 0.617 \\
\hline Employment status (employed) & -0.188 & 0.210 & -0.043 & -0.89 & 0.372 & -0.146 & 0.166 & -0.033 & -0.88 & 0.379 \\
\hline Income & -0.002 & 0.001 & -0.150 & -3.25 & 0.001 & -0.001 & 0.000 & -0.080 & -2.17 & 0.030 \\
\hline Comorbidities (yes) & -0.239 & 0.185 & -0.058 & -1.29 & 0.197 & -0.066 & 0.146 & -0.016 & -0.45 & $0.65 I$ \\
\hline BMI & 0.104 & 0.014 & 0.297 & 7.26 & $<0.001$ & 0.061 & 0.012 & 0.174 & 5.29 & $<0.001$ \\
\hline Diabetes duration (years) & 0.011 & 0.015 & 0.033 & 0.70 & 0.484 & 0.006 & 0.012 & 0.019 & 0.51 & 0.609 \\
\hline Treatment type (use Insulin) & 0.671 & 0.184 & 0.156 & 3.65 & $<0.001$ & 0.643 & 0.149 & 0.0149 & 4.31 & $<0.001$ \\
\hline Previous diabetes education (yes) & -0.385 & 0.262 & -0.059 & -1.47 & 0.141 & -0.240 & 0.206 & -0.037 & -1.16 & 0.245 \\
\hline Help with diabetes self-care (yes) & -0.289 & 0.172 & -0.070 & -1.68 & 0.093 & -0.168 & 0.137 & -0.041 & -1.22 & 0.222 \\
\hline Psychological support (yes) & -0.319 & 0.223 & -0.059 & -1.43 & 0.154 & 0.081 & 0.177 & 0.015 & 0.05 & 0.647 \\
\hline Psychological stress & 0.045 & 0.022 & 0.083 & 2.05 & 0.041 & 0.025 & 0.017 & 0.047 & 1.46 & 0.145 \\
\hline General diet & & & & & & -0.179 & 0.029 & -0.209 & -6.25 & $<0.001$ \\
\hline Specific diet & & & & & & -0.217 & 0.048 & -0.153 & -4.49 & $<0.001$ \\
\hline Exercise & & & & & & -0.616 & 0.060 & -0.363 & -10.3 & $<0.001$ \\
\hline Blood sugar testing & & & & & & -0.188 & 0.045 & -0.143 & -4.15 & $<0.001$ \\
\hline Foot care & & & & & & -0.017 & 0.034 & -0.018 & -0.519 & 0.604 \\
\hline $\mathrm{R}$ & 0.47 & & & & & 0.73 & & & & \\
\hline$R^{2}(\%)$ & 22.3 & & & & & 52.6 & & & & \\
\hline $\mathrm{F}$ & 10.25 & & & & $<0.001$ & 28.98 & & & & $<0.001$ \\
\hline$F$ for change in $R^{2}$ & & & & & & 63.52 & & & & $<0.001$ \\
\hline
\end{tabular}

Abbreviations: HbAlc, glycated hemoglobin; B, unstandardized coefficient; SE, standard error; $\beta$, standardized coefficient; BMI, body mass index.

study was conducted in different settings, in west-central Jordan, and with smaller sample size. ${ }^{36}$ Unsatisfactory compliance to diabetes self-care behaviors might be attributed to many potential cultural, social, financial, medical, and other barriers, not evaluated in the current study, that inhibited the participants' adherence to the recommended self-care activities. ${ }^{29}$ Most participants who reported to have never attended diabetes education sessions were low-income with a secondary education or less, which might explain their low level of self-care behaviors. Literature reported that attending diabetes education sessions and highereducation levels were significant predictors of self-care behaviors, and are associated with improved self-efficacy and diabetes care skills. ${ }^{28,55}$ Financial strains are major barriers in accessing diabetes care services, healthy foods, medications, and supplies, such as meter testing strips. ${ }^{28,56}$ Noncompliance can lead to suboptimal health outcomes, complications, death, and increased healthcare costs. Nurses and other healthcare professionals should plan and implement effective diabetes self-management education programs to promotr patients compliance to healthy lifestyles and self-care behaviors. 
According to study results, the least often performed self-care behaviors were exercise and BST, with the least compliance to participation in a specific exercise session and testing blood sugar as recommended. Other studies reported consistent findings. ${ }^{4,18,29-31}$ A possible reason for the unsatisfactory exercise levels is that $49.3 \%$ of the participants were above the age of 55 years, which is usually associated with a decline in health status and ability to perform exercises. The next low frequent self-care behaviors were dietary and foot care, with the least adherence being avoiding high-fat foods, inspecting the inside of shoes, and checking feet activities. Others reported similar poor dietary adherence and foot care among patients with T2DM. ${ }^{4,12,18,29,30}$ Interestingly, participants' adherence to a specific diet was higher than that of a general diet, which others reported. ${ }^{18,29}$ Consistently, Bawadi et al reported that diabetic patients in Jordan failed to achieve the recommended dietary guidelines for Arabs. Their poor glycemic control was attributed to their high consumption of sugar, carbohydrates, and sodium, and low consumption of polyunsaturated fatty acids. ${ }^{57}$

In addition, participants' adherence to feet washing was satisfactory, which is more likely to be performed frequently by Muslims as a religious practice. Other Jordanian studies reported similar findings. ${ }^{18,29,42}$ The most often performed and satisfactory behavior was taking medication, resembling results of other studies. ${ }^{12,18,29-31,33}$ These findings may imply that patients with T2DM were more likely to follow self-care behaviors that require the least lifestyle changes and efforts. ${ }^{29}$ However, in Jordan, food culture, traditional eating customs, and lack of culturally sensitive outdoor sports facilities are significant socio-cultural barriers that restrict diabetic patients' ability to engage in dietary management and physical activity. ${ }^{41}$ For example, not all communities in Jordan are amenable to physical exercise due to a lack of sidewalks, playgrounds, and fitness centers. Jordanian diabetic patients reported having exercise barriers, which were significant predictors of exercise duration. ${ }^{58}$ Higher score of barriers to regimen behavior was significantly associated with higher odds of poor glycemic control among Jordanian patients. ${ }^{16}$

Poor glycemic control among most of the participants indicates that taking medication alone is inadequate in ensuring good glycemic control. The participants depended more on medications and ignored the need to modify their lifestyle as diabetic patients, resulting in a loss of control over their blood glucose levels. For example, regular monitoring of blood sugar is essential in identifying their FBG level to adjust their diet, exercise frequency, and medication dose. Otherwise, their regular medication intake does not imply taking the proper dosage according to the required dose modification. This finding concurs with previous studies that found poor glycemic control among patients who had high adherence to medication, but low adherence to the dietary regimen, glucose monitoring, and physical exercise ${ }^{18,30,31,42}$ Our results indicate serious limitations in the methods utilized to promote diabetes self-care behaviors. Socio-cultural sensitive strategic interventions that focus on enhancing patients' lifestyle modifications are required to cultivate BST, exercise, foot care, and a dietary regimen as a part of their daily habits to promote the control of diabetes.

In addition, the findings showed that greater compliance to diabetes self-care behaviors was significantly associated with lower HbAlc levels, which is congruent with many previous studies. ${ }^{4,8,13,16-18,30,33}$ However, the most important independent predictor of glycemic control was exercise, followed by general diet, specific diet, and BST; greater adherence to these self-care behaviors was significantly associated with lower HbAlc levels. Literature reported a similar inverse association between HbA1c levels and exercise, ${ }^{8,10,11,18,33}$ diet, ${ }^{8,16,18,33}$ BST behaviors. ${ }^{8}$ Conversely, some studies reported that exercise, ${ }^{23,24,26,35}$ diet care, ${ }^{10,11,23,26}$ and BST were insignificant predictors of glycemic control. ${ }^{10,18,23,24,26,33}$ Furthermore, some studies found none of the diabetes self-care behaviors to be significant predictors of glycemic control, ${ }^{35,36}$ which can be explained by the presence of a strong family support system. ${ }^{9}$ For example, Qteishat and Ghananim could not detect the impact of diabetes self-care activities on glycemic control because most of the patients, who also reported adequate family support, had high adherence to self-care activities. ${ }^{36}$ Family provides financial and emotional support, homemade food, reassurance, and motivation for a patient to strictly follow a treatment regimen to improve their fight against diabetes-related stress and depression. ${ }^{59}$

In the current study, higher foot care adherence was significantly associated with lower HbAlc levels, while medication adherence was not. However, both foot care and medication adherence did not significantly contribute to the prediction of HbA1c levels, supporting other studies' findings of medication ${ }^{18,35,36}$ and foot care ${ }^{10,11,18,26,33,35,36}$ as insignificant predictors. Conversely, some studies reported medication as a significant predictor. ${ }^{10,23,24,26,33,34}$ Our findings indicated that the different self-care behaviors (healthy diet, BST, exercise) were independent of each other's behavior, and other sociodemographic and clinical factors, in glycemic control predictions. Diabetes education programs 
should focus on exercise, general and specific diet adherence, and BST behaviors to ensure they are practiced regularly and effectively to achieve optimal glycemic control among patients with T2DM.

\section{Sociodemographic and Clinical Factors and Their Contribution to Glycemic Control}

Besides self-care behaviors, some sociodemographic and clinical factors emerged as significant independent predictors of glycemic control. In the current study, higher monthly income, ${ }^{17,37}$ higher educational level, and being employed were associated with better glycemic control, consistent with many studies. ${ }^{4,11,16,22,41} 80 \%$ of participants with very low monthly income, 312.8 JD's (441.2 USD) per household, had 68.63 JD's (96.66 USD) per household member considering income to household size ratio. Most participants had secondary education or less and were unemployed. Literature reported financial strain as a significant barrier in diabetes self-care behaviors, particularly in accessing diabetes care, testing supplies, and healthy foods as recommended. ${ }^{28,56}$ Education level was also reported to influence diabetes care self-efficacy and skills. ${ }^{28,55}$ However, in the current study, low income was the only socioeconomic-independent predictor of poor glycemic control when considering diabetes self-care behaviors and other factors as predictors. This finding is consistent with a previous Jordanian study. ${ }^{17}$

In addition, among the clinical variables, the BMI and diabetes treatment type were independent predictors of glycemic control when simultaneously considered with diabetes self-care behaviors and confounding factors. Most study participants were obese or overweight, which is expected with their low level of physical activity; those with higher BMI (obesity) were more likely to have poor glycemic control compared to those with normal BMI, supporting previous findings. ${ }^{13,52}$ Many studies found BMI has associated with glycemic control but is not an independent predictor. ${ }^{15,16,18,24}$ Conversely, some studies reported that patients with normal BMI were more likely to have poor glycemic control than overweight and obese patients, which was explained by the disease process of diabetes where poor glycemic control is associated with weight loss. In contrast, improved glycemic control is associated with weight gain. ${ }^{23}$

Participants taking insulin treatments (alone or in combination with OHA) were more likely to have poor glycemic control than those receiving diabetic tablets only, supporting previous studies' findings. ${ }^{11,16,18,22,35}$ This result reflects more progressive diabetes and impairment of insulin action and/or secretion over time, making the response to glycemic control more difficult, and requiring more aggressive treatment. ${ }^{18,35}$ Therefore, possibly insulin was added as a treatment therapy for participants who had more severe T2DM and worsening glycemic control. However, the majority (92.7\%) of participants had poor glycemic control, possibly due to insulin therapy not being appropriately intensified. Alternatively, participants could lack the knowledge and skills required for optimal insulin management, such as the importance of BST for identifying their FBG level to adjust their medication dose, exercise, and diet. Accordingly, diabetes self-care education and behavioral interventions should enhance insulin uptake by patients' self-care behaviors by increasing their exercise levels, eating healthy low-fat foods, and BST to improve insulin uptake and achieve good glycemic control.

\section{Psychological Distress and Its Contribution for Glycemic Control}

Psychological distress was a significant independent predictor after controlling sociodemographic and clinical factors, though this significance disappeared when considered jointly with diabetes self-care behaviors. This finding concurs with prior studies. ${ }^{17,35}$ Many studies reported that as psychological distress tended to rise, participants' HbAlc levels rose accordingly. ${ }^{10,25,37,38,60}$ Also, mild to moderate depression was associated with inadequate diabetes self-care activities, ${ }^{9}$ including eating a healthy diet and $\mathrm{BST}^{40}$ Thus, the coexistence of depression and diabetes increases the burden and cost of diabetes management. ${ }^{25}$ High prevalence rates of depression (11.5-18.4-34.4\% of), anxiety (12.1-30-58.7\%), and stress (12.5-21.6) among patients with T2DM were reported; ${ }^{25,37,38}$ those with higher HbA1c were more likely to have psychological stress. ${ }^{25,38}$ In the current study, psychological support by family members was provided to $82.7 \%$ of the participants, yet a considerable proportion of participants had at least moderate $(30 \%)$ to severe levels $(17.5 \%)$ of psychological distress, which requires attention, through screening and treatment, from health care professionals to facilitate self-care management and glycemic control. Psychological interventions have a positive impact on coping with stress, self-care adherence, and metabolic control. ${ }^{61}$

In brief, diabetes self-care behaviors significantly contributed to the prediction of glycemic control, after controlling the effect of other factors. Higher levels of compliance with exercise, general (following a healthy eating plan) and 
specific diet (fruits, vegetables, and low-fat foods intake), and BST significantly predicted lower HbA1c levels. However, foot care and taking medication did not significantly contribute to the prediction of glycemic control. Moreover, high BMI, insulin therapy, and low monthly income were significantly associated with poor glycemic control. Some factors, such as diabetes-care help, previous diabetes-related education, educational level, and psychological stress, appear to be slightly negatively associated with $\mathrm{HbAlc}$ levels, which may be of practical importance despite the lack of statistical significance in the model; their values may be helpful for diabetic patients' glycemic control.

\section{Study Strengths and Limitations}

One of the strengths of this study is the utilization of valid and reliable SDSCA and PHQ-4 tools in the Arabic language. Also, the study assessed many variables that might affect glycemic control, which were not included in previous Jordanian studies. Although great care was taken to cut the effects of confounding variables, some factors might limit the generalizability of the results. Patients were chosen from hospitals in the same region to control the external factors affecting the control of diabetes; the study was conducted in just three governmental and teaching hospitals in northern Jordan, thus limiting the generalizability of the results. Also, the study used the convenience sampling method, in which the sample may not be representative of all diabetic patients in Jordan. Future studies should consider randomized samples with regions other than the north, where the study had been conducted. Further, this study used a cross-sectional design that did not examine the changes in diabetes self-care behaviors and their association with glycemic control over time, thus could not infer cause-and-effect relationships. Therefore, our study's "predict" term represents the association between study variables using regression analysis. Longitudinal research is required to validate and expand our findings.

In addition, data were collected by face-to-face structured interviews using self-report questionnaires. The recall and social desirability biases threatened the objectivity of participants' responses. Self-reporting bias is expected; some participants exaggerate their responses especially when asked sensitive questions like their salary rate and economic status. Over-reporting of socially desirable behaviors such as following healthy diets and eating fruits and vegetables is expected, leading to overestimation of satisfactory adherence. However, nearly $79 \%$ of participants had unsatisfactory self-care adherence, suggesting a small effect of overestimation. Self-report is the most practical method to measure health behavior. ${ }^{52}$

\section{Practical Implications}

These findings indicate that the status of glycemic control and diabetes self-care behavior among Jordanian patients with T2DM is alarmingly poor. The importance of diabetes self-care behaviors in controlling glycemic control suggests the necessity of designing cost-effective and socio-cultural sensitive strategic interventions to promote patients' self-care behaviors. Most participants never attended diabetes education sessions, representing an enormous need for patienttailored diabetes self-care educational programs, conducted regularly by qualified diabetes educators. Diabetes self-care education and behavioral interventions for Jordanian patients should focus on exercise, general and specific health diet, and blood sugar testing to ensure these behaviors are practiced regularly and effectively to achieve optimal glycemic control.

However, a wide range of individual, social, environmental, and cultural factors may affect patients' self-care behaviors. Therefore, counseling and supportive interventions are very important to provide suitable solutions tailored to individual needs. Further, community designing strategies are required to facilitate physical exercise levels by increasing culturally sensitive facilities and safe areas, such as safe playgrounds, sidewalks, and fitness centers at a low cost to overcome exercise barriers. ${ }^{58}$ Future studies are recommended to explore facilitator and barrier factors that affect patients' ability to manage their diabetes.

\section{Conclusion}

The prevalence of poor glycemic control is very high among patients with T2DM in northern Jordan, which was higher than that reported by previous studies from Jordan and other countries. The most important behavioral predictor of glycemic control was exercise, followed by general, specific diet, and BST. The least often performed self-care behaviors were exercise and BST. In contrast, medication was the most often performed behavior, indicating that patients with 
T2DM were more likely to follow self-care behaviors that require the least lifestyle changes. Likewise, BMI, treatment type, and income significantly contributed to the prediction of glycemic control. One-third of participants had moderate psychological distress which tended to slightly decrease their glycemic control, donating the need for regular psychological stress screening, and follow-up care.

\section{Acknowledgments}

This study was funded by the Faculty of Scientific Research/Jordan University of Science and Technology (Fund no. 333/ 2017). The authors would like to thank patients and institutions involved in the study.

\section{Disclosure}

The authors declare no conflicts of interest in this work.

\section{References}

1. World Health Organization. Classification of Diabetes Mellitus. Vol. 309. Geneva: World Health Organization; 2019. doi:10.1016/S0140-6736(77) 92735-0

2. International Diabetes Federation. IDF Diabetes Atlas. 9th ed. Brussels, Belgium: International Diabetes Federation; 2019. Available from: http:// www.diabetesatlas.org.

3. Ajlouni K, Batieha A, Jaddou H, et al. Time trends in diabetes mellitus in Jordan between 1994 and 2017. Diabet Med. 2019;36(9):1176-1182. doi:10.1111/DME.13894

4. Kayar Y, Ilhan A, Kayar NB, et al. Relationship between the poor glycemic control and risk factors, life style and complications. Biomed Res. 2017;28(4):1581-1586.

5. American Diabetes Association. 11. Microvascular complications and foot care: standards of Medical Care in Diabete-2020. Diabetes Care. 2020;43(Supplement1):S135-S151. doi:10.2337/dc20-S011

6. Lotfy M, Adeghate J, Kalasz H, Singh J, Adeghate E. Chronic complications of diabetes mellitus: a mini review. Curr Diabetes Rev. 2017;13 (1):3-10. doi:10.2174/1573399812666151016101622

7. Knight J, Andrade M, Nigam Y. Diabetes management. long term complications due to poor control. Nurs Times. 2017;113(4):45-48.

8. Bukhsh A, Khan TM, Nawaz MS, et al. Association of diabetes-related self-care activities with glycemic control of patients with type 2 diabetes in Pakistan. Patient Prefer Adherence. 2018;12:2377-2385. doi:10.2147/PPA.S177314

9. Hai AA, Iftikhar S, Latif S, Herekar F, Patel MJ. Diabetes self-care activities and their relation with glycemic control in patients presenting to The Indus Hospital, Karachi. Cureus. 2019;11(12). doi:10.7759/cureus.6297

10. Song X, Chen L, Zhang T, et al. Negative emotions, self-care activities on glycemic control in adults with type 2 diabetes: a cross-sectional study. Psychol Heal Med. 2020. doi:10.1080/13548506.2020.1799042

11. Ashur ST, Shah SA, Bosseri S, Fah TS, Shamsuddin K. Glycaemic control status among type 2 diabetic patients and the role of their diabetes coping behaviours: a clinic-based study in Tripoli, Libya. Libyan J Med. 2016;11:31086. doi:10.3402/ljm.v11.31086

12. AlQahtani AH, Alzahrani AS, Alzahrani SH, Alqahtani SM, AlOtaibi AF, Khan AA. Levels of practice and determinants of diabetes self-care in primary health care in Jeddah City, Saudi Arabia. Cureus. 2020;12(6). doi:10.7759/cureus. 8816

13. Oluma A, Abadiga M, Mosisa G, Etafa W. Magnitude and predictors of poor glycemic control among patients with diabetes attending public hospitals of Western Ethiopia. PLoS One. 2021;16(2):1-16. doi:10.1371/journal.pone.0247634

14. Haddad JA, Al Hyari MA, Al Momani MS, Al Omari AA, Ammari FL, Annabi FO. Baseline characteristics and treatment pattern of type 2 diabetes patients in Jordan: analysis from the DISCOVER patient population. Alexandria $J$ Med. 2020;56(1):51-55. doi:10.1080/ 20905068.2020.1747733

15. Al Hourani HM, Atoum MF. Factors associated with poor glycemic control among women with type 2 diabetes attending outpatient clinics in Jordan. Euromediterranean Biomed J. 2020;15(48):203-208. doi:10.3269/1970-5492.2020.15.48

16. Khattab M, Khader YS, Al-Khawaldeh A, Ajlouni K. Factors associated with poor glycemic control among patients with type 2 diabetes. J Diabetes Complications. 2010;24(2):84-89. doi:10.1016/j.jdiacomp.2008.12.008

17. Hammad S, Darawad M, Hourani E, Demeh W. Predictors of glycated hemoglobin among Jordanian diabetic patients. Iran J Public Heal. 2015;44 (11):1482-1491.

18. Al-Khawaldeh OA, Al-Hassan MA, Froelicher ES. Self-efficacy, self-management, and glycemic control in adults with type 2 diabetes mellitus. $J$ Diabetes Complications. 2012;26(1):10-16. doi:10.1016/j.jdiacomp.2011.11.002

19. Alzaheb R, Altemani A. The prevalence and determinants of poor glycemic control among adults with type 2 diabetes mellitus in Saudi Arabia. Diabetes Metab Syndr Obes Targets Ther. 2018;11:15-21. doi:10.2147/DMSO.S156214

20. Alhaiti AH, Senitan M, Dator WLT, et al. Adherence of type 2 diabetic patients to self-care activity: tertiary care setting in Saudi Arabia. J Diabetes Res. 2020;2020:1-7. doi:10.1155/2020/4817637

21. D'souza MS, Karkada SN, Venkatesaperumal R, Natarajan J. Self-care behaviours and glycemic control among adults with type 2 diabetes. GSTF J Nurs Heal Care. 2015;2(1). doi:10.5176/2345-718X_2.1.60

22. Kassahun T, Eshetie T, Gesesew H. Factors associated with glycemic control among adult patients with type 2 diabetes mellitus: a cross-sectional survey in Ethiopia Endocrine Disorders. BMC Res Notes. 2016;9(1):1-6. doi:10.1186/s13104-016-1896-7

23. Kamuhabwa AR, Charles E. Predictors of poor glycemic control in type 2 diabetic patients attending public hospitals in Dar Es Salaam. Drug Healthc Patient Saf. 2014;6:155-165. doi:10.1097/00005072-194804000-00006

24. Musenge EM, Michelo C, Mudenda B, Manankov A. Glycaemic control and associated self-management behaviours in diabetic outpatients: a hospital based observation study in Lusaka, Zambia. J Diabetes Res. 2016;2016. doi:10.1155/2016/7934654 
25. Kaur G, Tee GH, Ariaratnam S, Krishnapillai AS, China K. Depression, anxiety and stress symptoms among diabetics in Malaysia: a cross sectional study in an urban primary care setting. BMC Fam Pract. 2013;14(69):1-13. doi:10.1186/1471-2296-14-69

26. Osborn CY, Mayberry LS, Kim JM. Medication adherence may be more important than other behaviours for optimizing glycaemic control among low-income adults. J Clin Pharm Ther. 2016;41(3):256-259. doi:10.1111/jcpt.12360

27. Kolb L; Association of Diabetes Care and Education Specialists. An effective model of diabetes care and education: the ADCES7 self-care behaviorsTM. Sci Diabetes Self-Management Care. 2021;47(1):30-53. doi:10.1177/0145721720978154

28. Adu MD, Malabu UH, Malau-Aduli AEO, Malau-Aduli BS. Enablers and barriers to effective diabetes self-management: a multi-national investigation. PLoS One. 2019;14:e0217771. doi:10.1371/journal.pone.0217771

29. Albikawi ZF, Abuadas M. Diabetes self care management behaviors among Jordanian type two diabetes. Am Int J Contemp Res. 2015;5(3):87-95.

30. AlJohani KA, Kendall GE, Snider PD. Self-management practices among type 2 diabetes patients attending primary health-care centres in Medina, Saudi Arabia. East Mediterr Heal J. 2015;21(9):621-628. doi:10.26719/2015.21.9.621

31. Dos Santos SD, da Rocha MR, de Moura IH, et al. Self-care activities in people with type 2 diabetes mellitus. J Nurs UFPE line. 2019 ; $13: \mathrm{e} 241793$. doi:10.5205/1981-8963.2019.241793

32. Regina Baptista D, Wiens A, Pontarolo R, Regis L, Christine Torelli Reis W, Januário Correr C. The chronic care model for type 2 diabetes: a systematic review. Diabetol Metab Syndr. 2016;8(7). doi:10.1186/s13098-015-0119-z

33. Saumika MAR, Amarasekara TD, Jayasekara R. Diabetes self-care activities and glycaemic control among adults with type 2 diabetes in Sri Lanka: a cross-sectional study. J Biosci Med. 2019;7(5):99-111. doi:10.4236/jbm.2019.75013

34. Abebe SM, Berhane Y, Worku A, Alemu S, Mesfin N. Level of sustained Glycemic control and associated factors among patients with diabetes mellitus in Ethiopia: a hospital-based cross-sectional study. Diabetes Metab Syndr Obes Targets Ther. 2015;8:65-71. doi:10.2147/DMSO.S75467

35. Aga F, Dunbar SB, Kebede T, Higgins MK, Gary R. Relationships of diabetes self-care behaviours to glycaemic control in adults with type 2 diabetes and comorbid heart failure. Nurs Open. 2020;7(5):1453-1467. doi:10.1002/nop2.517

36. Qteishat RR, Al Ghananim AR. Comprehensive assessment of variables affecting metabolic control in patients with type 2 diabetes mellitus in Jordan. Diabetes Metab Syndr. 2015;10(1 Suppl 1):S56-S59. doi:10.1016/j.dsx.2015.09.014

37. Bener A, Ozturk M, Yildirim E. Association between depression, anxiety and stress symptoms and glycemic control in diabetes mellitus patients. Int J Clin Endocrinol. 2017;1(1):1-7.

38. Camara A, Baldé NM, Enoru S, Bangoura JS, Sobngwi E, Bonnet F. Prevalence of anxiety and depression among diabetic African patients in Guinea: association with HbA1c levels. Diabetes Metab. 2015;41(1):62-68. doi:10.1016/j.diabet.2014.04.007

39. Al-Hayek AA, Robert AA, Alzaid AA, et al. Association between diabetes self-care, medication adherence, anxiety, depression, and glycemic control in type 2 diabetes. Saudi Med J. 2012;33(6):681-683. doi:10.1016/j.jallcom.2011.06.011

40. Al-Amer RM, Sobeh MM, Zayed AA, Al-Domi HA. Depression among adults with diabetes in Jordan: risk factors and relationship to blood sugar control. J Diabetes Complications. 2011;25(4):247-252. doi:10.1016/j.jdiacomp.2011.03.001

41. Al-Sahouri A, Merrell J, Snelgrove S. Barriers to good glycemic control levels and adherence to diabetes management plan in adults with type-2 diabetes in Jordan: a literature review. Patient Prefer Adherence. 2019;13:675-693. doi:10.2147/PPA.S198828

42. Madae'en S, Elayeh E, Akour A, Alqhaiwi T, Shaggour B, Madain R. Diabetes knowledge, medication adherence, and glycemic control among diabetic patients: a cross-sectional study in Jordan ARTICLE INFO. J Appl Pharm Sci. 2020;10(4):41-046. doi:10.7324/JAPS.2020.104007

43. Jenkins DG, Quintana-Ascencio PF. A solution to minimum sample size for regressions. PLoS One. 2020;15(2):e0229345. doi:10.1371/JOURNAL. PONE.0229345

44. World Healt Organization Expert Consultation. Appropriate body-mass index for Asian populations and its implications for policy and intervention strategies. Lancet. 2004;363(9403):157-163. doi:10.1016/S0140-6736(03)15268-3

45. American Diabetes Association. Glycemic targets: standards of medical care in diabetes-2020. Diabetes Care. 2020;43(1):S66-S76. doi:10.2337/ dc20-S006

46. Kroenke K, Spitzer RL, Williams JBW, Lowe B. An ultra-brief screening scale for anxiety and depression: the PHQ-4. Psychosomatics. 2009;50 (6):613-621. doi:10.1176/appi.psy.50.6.613

47. Joseph R, Lucca JM, Alshayban D, Alshehry YA. The immediate psychological response of the general population in Saudi Arabia during COVID-19 pandemic: a cross-sectional study. J Infect Public Health. 2021;14:1-8. doi:10.1016/j.jiph.2020.11.017

48. Löwe B, Wahl I, Rose M, et al. A 4-item measure of depression and anxiety: validation and standardization of the Patient Health Questionnaire-4 (PHQ-4) in the general population. J Affect Disord. 2010;122(1-2):86-95. doi:10.1016/j.jad.2009.06.019

49. AlJohani KA, Kendall GE, Snider PD. Psychometric evaluation of the summary of diabetes self-care Activities-Arabic (SDSCA-Arabic): translation and analysis process. J Transcult Nurs. 2016;27(1):65-72. doi:10.1177/1043659614526255

50. Sukkarieh-Haraty O, Howard E. Psychometric properties of the Arabic version of the EUROPEP questionnaire. Res Theory Nurs Pract. 2016;30 (1):60-69. doi:10.1891/1541-6577.30.1.60

51. Toobert DJ, Hampson SE, Glasgow RE. The summary of diabetes self-care: results from 7 studies and a revised scale. Diabetes Care. 2000;23 (7):943-950. doi:10.2337/diacare.23.7.943

52. Tharek Z, Ramli AS, Whitford DL, et al. Relationship between self-efficacy, self-care behaviour and glycaemic control among patients with type 2 diabetes mellitus in the Malaysian primary care setting. BMC Fam Pract. 2018;19(1). doi:10.1186/s12875-018-0725-6

53. Zhao -F-F, Katajisto J, Leino-Kilpi H, Leino-Kilpi H. The association of diabetes-related self-care activities with perceived stress, anxiety, and fatigue: a cross-sectional study. Patient Prefer Adherence. 2018;12:1677-1686. doi:10.2147/PPA.S169826

54. Banerji MA, Dunn JD. Impact of glycemic control on healthcare resource utilization and costs of type 2 diabetes: current and future pharmacologic approaches to improving outcomes. Am Heal Drug Benefits. 2013;6(7):382-392.

55. Amer FAM, Mohamed MS, Elbur AI, Abdelaziz SI, Elrayah ZAB. Influence of self-efficacy management on adherence to self-care activities and treatment outcome among diabetes mellitus type 2 sudanese patients. Pharm Pract. 2018;16(4). doi:10.18549/PharmPract.2018.04.1274

56. Adhikari M, Devkota HR, Cesuroglu T. Barriers to and facilitators of diabetes self-management practices in Rupandehi, Nepal-multiple stakeholders' perspective. BMC Public Health. 2021;21(1269):1-18. doi:10.1186/s12889-021-11308-4

57. Bawadi H, Abu-Jamous D, Tayyem RF. Evaluation of the dietary pattern of patients with type 2 diabetes in Northern Jordan: an inconvenient truth! Int J Diabetes Dev Ctries. 2014;34(3):134-138. doi:10.1007/s13410-013-0149-8 
58. Darawad MW, Mosleh S, Khalil AA, et al. Investigating physical exercise among Jordanians with diabetes mellitus. Health. 2016;8(2):181-189. doi:10.4236/HEALTH.2016.82021

59. Ahmed Z, Yeasmeen F. Active family participation in diabetes self-care: a commentary. Diabetes Manag. 2016;6(5):104-107.

60. Bener A. High prevalence of depression, anxiety and stress symptoms among diabetes mellitus patients. Open Psychiatr J. 2011;5(1):5-12. doi: $10.2174 / 1874354401105010005$

61. Aljawarneh YM, Al-Qaissi NM, Ghunaim HY. Psychological interventions for adherence, metabolic control, and coping with stress in adolescents with type 1 diabetes: a systematic review. World J Pediatr. 2020;16(5):456-470. doi:10.1007/S12519-020-00352-6

\section{Publish your work in this journal}

Patient Preference and Adherence is an international, peer-reviewed, open access journal that focusing on the growing importance of patient preference and adherence throughout the therapeutic continuum. Patient satisfaction, acceptability, quality of life, compliance, persistence and their role in developing new therapeutic modalities and compounds to optimize clinical outcomes for existing disease states are major areas of interest for the journal. This journal has been accepted for indexing on PubMed Central. The manuscript management system is completely online and includes a very quick and fair peer-review system, which is all easy to use. Visit http://www.dovepress.com/testimonials.php to read real quotes from published authors.

Submit your manuscript here: https://www.dovepress.com/patient-preference-and-adherence-journal 